Abstract ID: 120

\title{
Production of monoclonal antibodies to immunogenic peptides of $\omega$-glaidin/c-hordein and rye-secalin for gluten free food screening
}

\author{
Widya Abd Wahab ${ }^{\mathrm{a}}$ | H Julia Ellis ${ }^{\mathrm{b}}$ | Paul J Ciclitira ${ }^{\mathrm{b}}$ \\ ${ }^{a}$ Kulliyyah of Science, International Islamic University Malaysia \\ ${ }^{b}$ King's College London
}

Introduction: The $\omega$-glaidin/c-hordein (QPFPQPEQPFPW) and rye-secalin (QPFPQPQQPIPQ) peptides have previously demonstrated immunogenicity in sensitised coeliac T cell lines. The development of monoclonal antibody to those immunogenic peptides is presented with a view of developing an improved enzyme linked immunosorbent assay (ELISA) as a reliable tool to screen the safety of foods specialised for coeliac disease (CD) patients. Methods: Balb/C mice were fed with gluten free food. The immunogens were conjugated to purified tuberculin protein derivative (PPD) with glutaraldehyde and emulsified in Freund's adjuvant. The employed immunization schedule included 3-5 weeks intervals, followed by a final intravenous injection without adjuvant, 3-4 days prior to fusion. Results: The antibody produced was IgM rather than IgG, although the hybridoma was successfully generated. The IgM class antibody is known to be relatively unstable, so could not be used in kits designed for food screening. Conclusions: The immunogenic peptides could possibly be used to raise monoclonal antibodies for gluten screening. However, a single booster injection might be insufficient to stimulate the spleen directly or the mice should be immunized with higher concentration of immunogen. This could be improved by including multiple booster administrations and increasing the dose.

KEYWORDS: coeliac disease (CD), monoclonal antibody (MAb), gluten, -glaidin/c-hordein and ryesecalin peptides 